\title{
Hypoglycemic effects of glabridin, a polyphenolic flavonoid from licorice, in an animal model of diabetes mellitus
}

\author{
FEIHUA WU, ZHIGUI JIN and JIAN JIN \\ Department of Pharmacy, Ninth People's Hospital, School of Medicine, \\ Shanghai Jiao Tong University, Huangpu, Shanghai 200011, P.R. China
}

Received October 17, 2012; Accepted January 11, 2013

DOI: $10.3892 / \mathrm{mmr} .2013 .1330$

\begin{abstract}
The present study was designed to investigate the hypoglycemic effects of glabridin from licorice in an animal model of diabetes mellitus (DM). Male Kunming mice were used to induce DM using streptozotocin (STZ). After confirmation of the diabetic state, the mice were randomly divided into six groups of 10 animals each: normal control (NC), diabetic control (DC), diabetic + low-dose glabridin treatment (DLG) (glabridin, $10 \mathrm{mg} / \mathrm{kg}$ ), diabetic + medium-dose glabridin treatment (DMG) (glabridin, $20 \mathrm{mg} / \mathrm{kg}$ ), diabetic + high-dose glabridin treatment (DHG) (glabridin, $40 \mathrm{mg} / \mathrm{kg}$ ) and diabetic + glyburide treatment group (DG) (glyburide, $4 \mathrm{mg} / \mathrm{kg}$ ). Each treatment was continued daily for 28 days, and then body weight, fasting blood glucose (FBG), glucose tolerance, superoxide dismutase (SOD) and malondialdehyde (MDA) were measured. The data obtained showed that glabridin significantly increased body weight, glucose tolerance and SOD activities in the liver, kidney and pancreas, while decreasing FBG levels and MDA content in the liver, kidney and pancreas. These results demonstrated that glabridin possesses hypoglycemic effects.
\end{abstract}

\section{Introduction}

Diabetes mellitus (DM) is a group of metabolic disorders with different underlying etiologies. It is characterized by absolute or relative deficiencies in insulin secretion and/or insulin action associated with chronic hyperglycemia and disturbances of carbohydrate, lipid and protein metabolism. As a consequence of the metabolic derangements in diabetes, various complications develop including macro- and microvascular dysfunctions $(1,2)$. The management of diabetes is

Correspondence to: Dr Zhigui Jin, Department of Pharmacy, Ninth People's Hospital, School of Medicine, Shanghai Jiao Tong University, 639 Zhizaoju Road, Huangpu, Shanghai 200011, P.R. China

E-mail: wufeihua1961@126.com; wufeihua1961@yahoo.com

Key words: hypoglycemic, glabridin, diabetes mellitus, mouse considered a global problem. Modern drugs, including insulin and other hyperglycemic agents such as biguanides and sulphonylureas control the blood glucose level only when they are regularly administered, although these treatments are laborious and have several disadvantages including hypoglycemia and obesity (3). Therefore the identification of efficacious agents with less severe side-effects is crucial. Over the past few decades, traditional Chinese medicine has played a key role in the therapy of DM and its complications $(4,5)$. Based on a large number of chemical and pharmacological research studies, numerous bioactive compounds have been found in Chinese medicinal plants for the treatment of diabetes. These compounds include polysaccharides, terpenoids, flavonoids, sterols and alkanoids (6-10).

Licorice is the root and stolon of the Glycyrrhiza plant, which belongs to the family Leguminosae. This plant has been medicinally used for $>4,000$ years (11). It is a Chinese herb commonly used as an expectorant and to arrest coughing, reduce fever, comfort the stomach, alleviate urgency and potentiate the effects of various other herbs (12). Licorice has been reported to attenuate free radical-induced oxidative damage in the kidney, prevent carcinogenesis induced by toxicants or hormones and also has a significant hepatoprotective activity (13-15). Licorice contains flavonoids and triterpenoids (15). Glabridin (Fig. 1), a polyphenolic flavonoid, is a main active component in licorice, which has been reported to exhibit multiple pharmacological activities, such as cytotoxic, antimicrobial, anti-fatigue, estrogenic and anti-proliferative activity against human breast cancer cells (16). It also affects melanogenesis, inflammation, low-density lipoprotein oxidation and protection of mitochondrial functions from oxidative stress (17). However, there is a limited number of studies on the effect of glabridin on DM at present. Thus, the aim of this study was to investigate the hypoglycemic effects of glabridin from licorice in an animal model of DM.

\section{Materials and methods}

Reagents. Glabridin (purity $>99 \%$ by HPLC analysis) was purchased from Shaanxi Langrun Biotechnology Co., Ltd. (Xi'an, China). Streptozotocin (STZ) was purchased from Sigma-Aldrich (St. Louis, MO, USA). Glyburide (glibenclamide) was purchased from Shanxi Sanjin Pharmaceutical Co., Ltd. (Taiyuan, China). Glucose kit was purchased from 
<smiles>CC1(C)C=Cc2c(ccc3c2OCC(c2ccc(O)cc2O)C3)O1</smiles>

Figure 1. Chemical structure of glabridin.

Biosino Biotechnology and Science, Inc. (Beijing, China). Superoxide dismutase (SOD) and malondialdehyde (MDA) kits were purchased from Jiancheng Bioengineering Institute (Nanjing, China). Other chemicals were obtained from local sources and were of analytical grade.

Animals. Male Kunming mice (2 months old, 18-22-g body weight) were obtained from the Shanghai Center of Experimental Animals (Shanghai, China). The animals were housed under standard environmental conditions $\left(22-25^{\circ} \mathrm{C}\right.$, humidity $60-70 \%, 12$-h light/dark cycle) with free access to standard diet and water ad libitum. The mice used in this study were processed in accordance with the UK Animals (Scientific Procedures) Act 1986 and associated guidelines. The experimental protocol was approved by the Shanghai Jiao Tong University Animal Care and Use Committee (Shanghai, China).

Induction of diabetes mellitus. The mice were fasted for $16 \mathrm{~h}$ prior to the induction of DM. STZ was freshly prepared in 0.1-mol/1 citrate buffer solution ( $\mathrm{pH} 4.5)$ and was intraperitoneally injected into mice with a single dose of $60 \mathrm{mg} / \mathrm{kg}$. DM was confirmed by the measurement of blood glucose from the tail vein $72 \mathrm{~h}$ after injection of STZ. Mice with a blood glucose level $>11.0 \mathrm{mmol} / 1$, as well as polydipsia, polyuria and polyphagia were selected for the experiment (18).

Experimental design. Following confirmation of the diabetic state, the mice were randomized into six groups of 10 animals each: i) normal control group (NC), non diabetic mice administered $0.5 \mathrm{ml}$ of $0.5 \%$ Tween- 80 solution; ii) diabetic control group (DC), diabetic mice administered $0.5 \mathrm{ml}$ of $0.5 \%$ Tween-80 solution; iii) diabetic + low-dose glabridin treatment group (DLG), diabetic mice administered glabridin $(10 \mathrm{mg} / \mathrm{kg})$ in $0.5 \mathrm{ml} 0.5 \%$ Tween- 80 solution; iv) diabetic + medium-dose glabridin treatment group (DMG), diabetic mice administered glabridin $(20 \mathrm{mg} / \mathrm{kg})$ in $0.5 \mathrm{ml} 0.5 \%$ Tween- 80 solution; v) diabetic + high-dose glabridin treatment group (DHG), diabetic mice administered glabridin $(40 \mathrm{mg} / \mathrm{kg})$ in $0.5 \mathrm{ml}$ $0.5 \%$ Tween- 80 solution and vi) diabetic + glyburide treatment group (DG), diabetic mice administered $0.5 \mathrm{ml}$ of $0.5 \%$ Tween- 80 solution containing glyburide $(4 \mathrm{mg} / \mathrm{kg})$.

Each treatment was continued daily for 28 days. Fasting blood glucose (FBG) levels were measured once every week. Blood was collected from the tip of the tail vein (starting from 9:00 a.m.) after a 12- to 14-h overnight fast. At the same time, body weights of mice were measured. An oral glucose tolerance test (OGTT) was performed on the last day of treatment

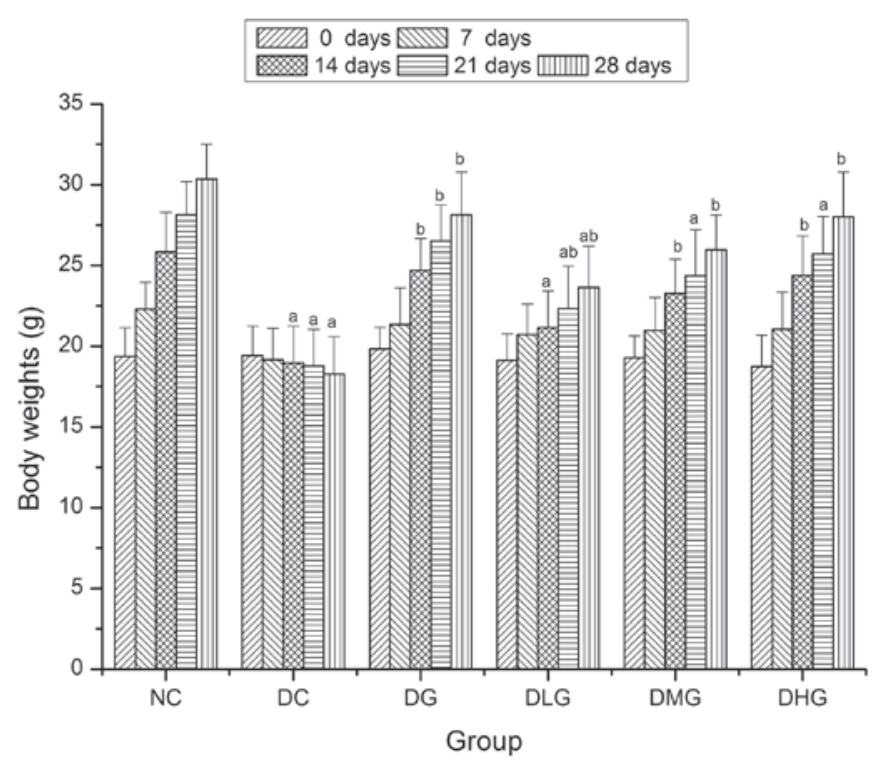

Figure 2. Effect of glabridin on the body weight of mice. Data are presented as the means $\pm \mathrm{SD}$. ${ }^{\mathrm{a}} \mathrm{P}<0.05$ when compared with the $\mathrm{NC}$ group; ${ }^{\mathrm{b}} \mathrm{P}<0.05$ when compared with the DC group. NC, normal control; DC, diabetic control; DG, diabetic + glyburide treatment; DLG, diabetic + low-dose glabridin treatment; DMG, diabetic + medium-dose glabridin treatment; DHG, diabetic + high-dose glabridin treatment.

after overnight fasting. Blood was collected at $0,30,60$ and $120 \mathrm{~min}$ after an oral glucose load of $3.0 \mathrm{~g} / \mathrm{kg}$ of body weight. Following completion of the experiment, the mice were sacrificed by cervical decapitation. The liver, kidney and pancreas were dissected out, washed in ice-cold saline, and homogenized in Tris- $\mathrm{HCl}$ buffer. Supernatant fractions of liver homogenate were used to measure SOD activity and MDA content.

Statistical analysis. Results were presented as the means \pm standard deviation (SD). The data were evaluated by means of an analysis of variance (ANOVA:MANOVA), using the Newman-Keuls test. Differences with a value of $\mathrm{P}<0.05$ were considered to indicate a statistically significant difference.

\section{Results}

Effect of glabridin on the body weight of mice. Prior to the experiment, the body weights were not significantly different among all the groups ( $\mathrm{P}>0.05)$ (Fig. 2). After 14 days, the body weights of the mice in the DG, DMG and DHG groups were significantly increased when compared with the DC group $(\mathrm{P}<0.05)$, while the body weights of mice in the DHG group were increased, although not significantly $(\mathrm{P}>0.05)$. After 28 days, the body weights of the mice in the DG, DLG, DMG and DHG groups were significantly increased when compared with the DC group $(\mathrm{P}<0.05)$, while the body weights of the mice in the DLG group remained significantly decreased when compared with the $\mathrm{NC}$ group $(\mathrm{P}<0.05)$.

Effect of glabridin on FBG levels of mice. As shown in Fig. 3, FBG levels in the NC group remained constant and were significantly decreased as compared with the diabetic groups (DC, DG, DLG, DMG and DHG) during the experimental period $(\mathrm{P}<0.05)$. After 7 days, FBG levels in the diabetic treat- 


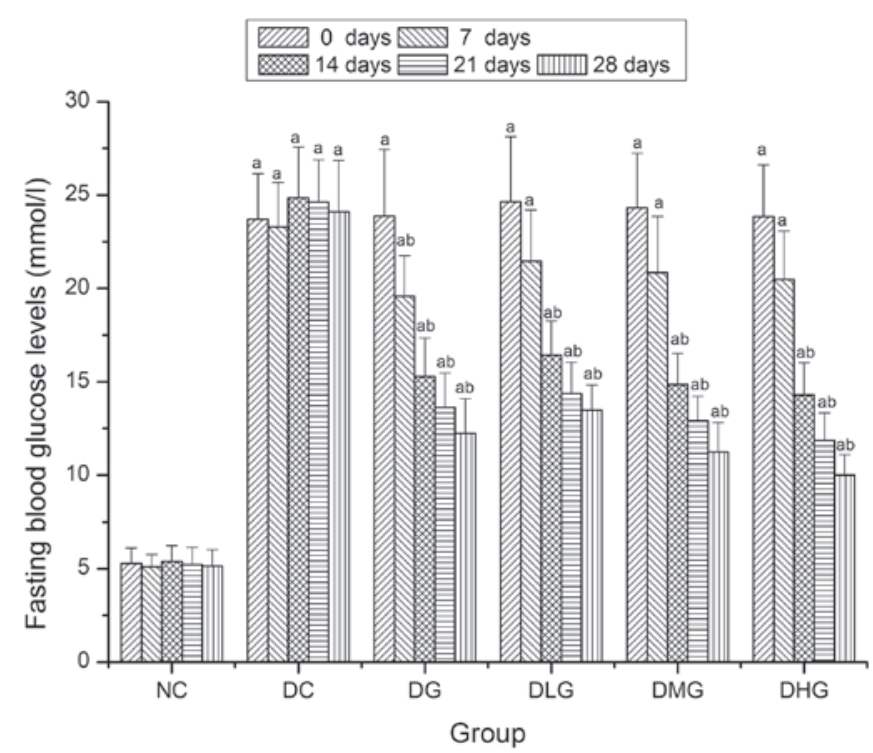

Figure 3. Effect of glabridin on fasting blood glucose (FBS) levels of mice. Data are presented as the means $\pm \mathrm{SD}$. ${ }^{a} \mathrm{P}<0.05$ when compared with the NC group; ${ }^{\mathrm{b}} \mathrm{P}<0.05$ when compared with the $\mathrm{DC}$ group. NC, normal control; DC, diabetic control; DG, diabetic + glyburide treatment; DLG, diabetic + low-dose glabridin treatment; DMG, diabetic + medium-dose glabridin treatment; DHG, diabetic + high-dose glabridin treatment.

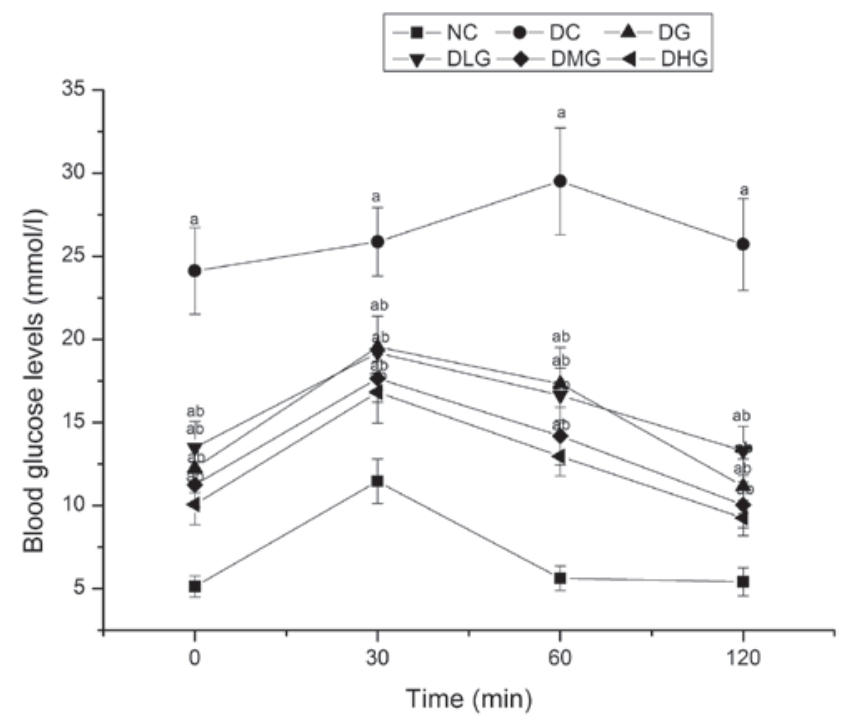

Figure 4. Effect of glabridin on glucose tolerance of mice. Data are presented as the means $\pm \mathrm{SD}$. ${ }^{\mathrm{a}} \mathrm{P}<0.05$ when compared with the $\mathrm{NC}$ group; ${ }^{\mathrm{b}} \mathrm{P}<0.05$ when compared with the DC group. NC, normal control; DC, diabetic control; DG, diabetic + glyburide treatment; DLG, diabetic + low-dose glabridin treatment; DMG, diabetic + medium-dose glabridin treatment; DHG, diabetic + high-dose glabridin treatment.

ment groups (DG, DLG, DMG and DHG) showed a decreasing trend. After 28 days, FBG levels in the DG, DLG, DMG and DHG groups were significantly decreased as compared with the DC group $(\mathrm{P}<0.05)$, being 196.9, 178.8, 214.6 and $240.9 \%$ lower, respectively.

Effect of glabridin on glucose tolerance of mice. Blood glucose levels in the DG, DLG, DMG and DHG groups were significantly decreased when compared with the DC groups at

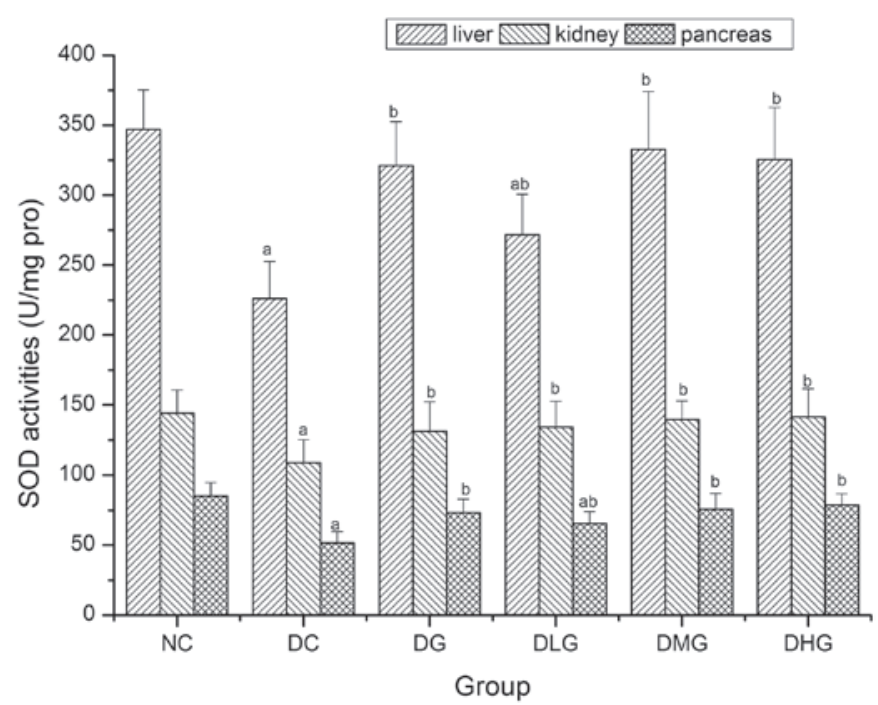

Figure 5. Effect of glabridin on superoxide dismutase (SOD) activities in the liver, kidney and pancreas of mice. Data are presented as the means \pm SD. ${ }^{a} \mathrm{P}<0.05$ when compared with the $\mathrm{NC}$ group; ${ }^{\mathrm{b}} \mathrm{P}<0.05$ when compared with the DC group. NC, normal control; DC, diabetic control; DG, diabetic + glyburide treatment; DLG, diabetic + low-dose glabridin treatment; DMG, diabetic + medium-dose glabridin treatment; DHG, diabetic + high-dose glabridin treatment.

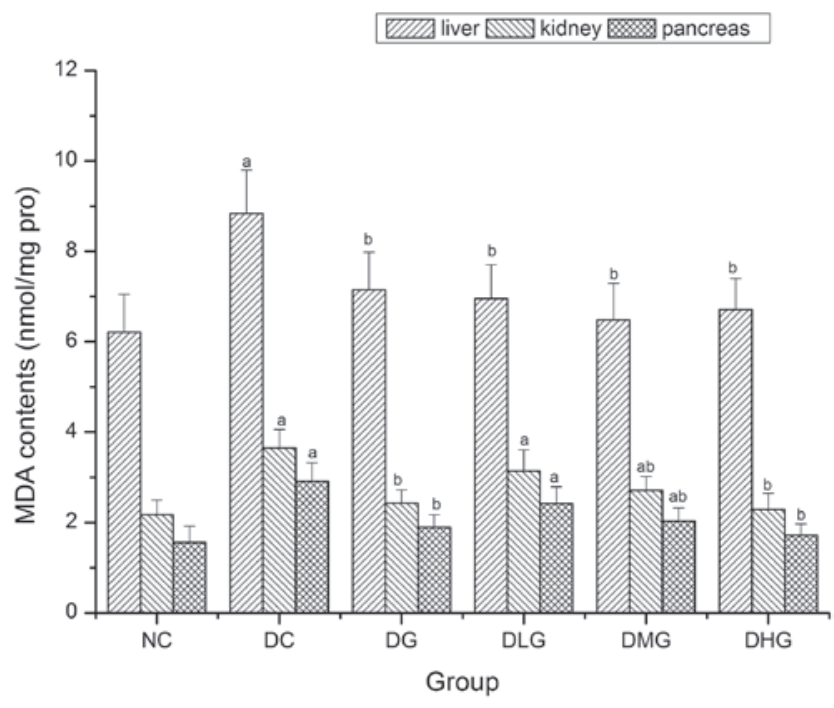

Figure 6. Effect of glabridin on malondialdehyde (MDA) contents in the liver, kidney and pancreas of mice. Data are presented as the means \pm SD. ${ }^{\mathrm{a}} \mathrm{P}<0.05$ when compared with the $\mathrm{NC}$ group; ${ }^{\mathrm{b}} \mathrm{P}<0.05$ when compared with the DC group. NC, normal control; DC, diabetic control; DG, diabetic + glyburide treatment; DLG, diabetic + low-dose glabridin treatment; DMG, diabetic + medium-dose glabridin treatment; DHG, diabetic + high-dose glabridin treatment.

different time intervals $(0,30,60$ and $120 \mathrm{~min})(\mathrm{P}<0.05)$, while they remained significantly increased when compared with the NC group $(\mathrm{P}<0.05)$ (Fig. 4).

Effect of glabridin on SOD activities in the liver, kidney and pancreas of mice. As shown in Fig. 5, when compared with the DC group, SOD activities in the liver, kidney and pancreas were significantly increased in the DG, DLG, DMG and DHG groups $(\mathrm{P}<0.05)$, while SOD activities in the liver and 
pancreas in the DLG group were still significantly decreased when compared with the NC group $(\mathrm{P}<0.05)$.

Effect of glabridin on MDA contents in the liver, kidney and pancreas of mice. MDA contents in the liver, kidney and pancreas were significantly decreased in the DG, DMG and DHG groups when compared with the DC group $(\mathrm{P}<0.05)$ (Fig. 6). In the DLG group, MDA content in the liver was significantly decreased $(\mathrm{P}<0.05)$ when compared with the DC group, and MDA contents in the kidney and pancreas were decreased, although not significantly $(\mathrm{P}<0.05)$.

\section{Discussion}

STZ (N-nitroso derivative of glucosamine) is a broadspectrum antibiotic extracted from Streptomyces achromogenes. It is a pancreatic $\beta$-cell toxin that induces rapid and irreversible necrosis of $\beta$-cells and is widely used to induce DM in experimental animal models $(19,20)$. STZ-induced DM is characterized by severe loss in body weight, which may be due to degradation of structural proteins since they are known to contribute to body weight (21). In this study, a significant body weight loss was observed in the DC group and significant improvement of body weight was observed in the glabridin treatment groups (DLP, DMP and DHP). This finding may be due to the ability of glabridin to reduce hyperglycemia.

$\mathrm{DM}$ is a serious chronic disease. Effective blood glucose control is the key to preventing or reversing diabetic complications and improving quality of life in patients with diabetes $(22,23)$. In the present study, STZ-induced diabetic mice presented obvious hyperglycemic symptoms, while glabridin produced a significant decrease in FBG levels in diabetic mice. In addition, glucose tolerance also improved significantly after glabridin treatment. These results indicated that glabridin possesses hypoglycemic effects and that the $40-\mathrm{mg} / \mathrm{kg}$ dose of the glabridin exerted a better effect when compared to doses of 10 or $20 \mathrm{mg}$.

Previous studies have shown that reactive oxygen species (ROS) and lipid peroxidation are important in the pathogenesis of DM and its complications (24-26). An imbalance between ROS generation and the reduced activity of antioxidant defenses or both of these phenomena lead to oxidative stress. Hyperglycemia is a cause of oxidative stress in diabetic patients and reduces the capacity of the endogenous antioxidant defense system via the production of several reducing sugars (through glycolysis and the polyol pathway) (26). In the present study, glabridin significantly increased SOD activities, while decreasing MDA contents in the liver, kidney and pancreas. Therefore, it may be concluded that the antioxidative activities of glabridin in STZ-induced diabetic mice, at least in part, may be related to hypoglycemic effects.

In summary, the present study has demonstrated that glabridin possesses hypoglycemic effects. The $40-\mathrm{mg} / \mathrm{kg}$ dose of glabridin exerted a better effect when compared to doses of 10 or $20 \mathrm{mg}$. Further pharmacological and biochemical investigations would clearly elucidate the mechanism of action and would be beneficial in investigating the role of glabridin as a therapeutic target in diabetes treatment research.

\section{Acknowledgements}

This study was supported by grants from the Research Project of Science and Technology of Shanghai Jiao Tong University (Shanghai, China).

\section{References}

1. El-Alfy AT, Ahmed AA and S Fatani AJ: Protective effect of red grape seeds proanthocyanidins against induction of diabetes by alloxan in rats. Pharmacol Res 52: 264-270, 2005.

2. Li F, Tang H, Xiao F, et al: Protective effect of salidroside from Rhodiolae radix on diabetes-induced oxidative stress in mice. Molecules 16: 9912-9924, 2011.

3. Zhang ZF, Lv GY, Pan HJ, et al: Antidiabetic activities of ethanol extract of dry matters of culture broth of Coriolus versiolor in submerged culture. Braz Arch Biol Technol 54: 701-708, 2011.

4. Li WL, Zheng HC, Bukuru J and De Kimpe N: Natural medicines used in the traditional Chinese medical system for therapy of diabetes mellitus. J Ethnopharmacol 92: 1-21, 2004.

5. Tang LQ, Wei W, Chen LM and Liu S: Effects of berberine on diabetes induced by alloxan and a high-fat/high-cholesterol diet in rats. J Ethnopharmacol 108: 109-115, 2006.

6. Luo Q, Cai Y, Yan J, et al: Hypoglycemic and hypolipidemic effects and antioxidant activity of fruit extracts from Lycium barbarum. Life Sci 76: 137-149, 2004.

7. Lü H, Chen J, Li WL, et al: Hypoglycemic effect of the total flavonoid fraction from folium Eriobotryae. Phytomedicine 16: 967-971, 2009.

8. Liu Z, Wang LJ, Li X, et al: Hypoglycemic effects of malonyl-ginsenosides extracted from roots of Panax ginseng on streptozotocin-induced diabetic mice. Phytother Res 23: 1426-1430, 2009.

9. Qi LW, Liu EH, Chu C, et al: Anti-diabetic agents from natural products - an update from 2004 to 2009. Curr Top Med Chem 10: 434-457, 2010

10. Patel MB and Mishra S: Hypoglycemic activity of alkaloidal fraction of Tinospora cordifolia. Phytomedicine 18: 1045-1052, 2011.

11. Tian ML, Yan HY and Row KH: Simultaneous extraction and separation of liquiritin, glycyrrhizic acid, and glabridin from licorice root with analytical and preparative chromatography. Biotechnol Bioprocess Eng 13: 671-676, 2008.

12. Sabbioni C, Mandrioli R, Ferranti A, et al: Separation and analysis of glycyrrhizin, 18beta-glycyrrhetic acid and 18alpha-glycyrrhetic acid in liquorice roots by means of capillary zone electrophoresis. J Chromatogr A 1081: 65-71, 2005.

13. Mori H, Niwa K, Zheng Q, et al: Cell proliferation in cancer prevention; effects of preventive agents on estrogen-related endometrial carcinogenesis model and on an in vitro model in human colorectal cells. Mutat Res 480-481: 201-207, 2001.

14. Yokozawa T, Cho EJ, Rhyu DY, et al: Glycyrrhizae Radix attenuates peroxynitrite-induced renal oxidative damage through inhibition of protein nitration. Free Radic Res 39: 203-211, 2005.

15. Lee JY, Lee JH, Park JH, et al: Liquiritigenin, a licorice flavonoid, helps mice resist disseminated candidiasis due to Candida albicans by Th1 immune response, whereas liquiritin, its glycoside form, does not. Int Immunopharmacol 9: 632-638, 2009.

16. Shang H, Cao S, Wang J, et al: Glabridin from Chinese herb licorice inhibits fatigue in mice. Afr J Tradit Complement Altern Med 7: 17-23, 2009.

17. Choi EM: The licorice root derived isoflavan glabridin increases the function of osteoblastic MC3T3-E1 cells. Biochem Pharmacol 70: 363-368, 2005

18. Sharma S, Kulkarni SK, Agrewala JN and Chopra K: Curcumin attenuates thermal hyperalgesia in a diabetic mouse model of neuropathic pain. Eur J Pharmacol 536: 256-261, 2006.

19. Hwang HJ, Kim SW, Lim JM, et al: Hypoglycemic effect of crude exopolysaccharides produced by a medicinal mushroom Phellinus baumii in streptozotocin-induced diabetic rats. Life Sci 76: 3069-3080, 2005.

20. Hayashi K, Kojima R and Ito M: Strain differences in the diabetogenic activity of streptozotocin in mice. Biol Pharm Bull 29: 1110-1119, 2006. 
21. Sarkhail P, Rahmanipour S, Fadyevatan S, et al: Antidiabetic effect of Phlomis anisodonta: effects on hepatic cells lipid peroxidation and antioxidant enzymes in experimental diabetes. Pharmacol Res 56: 261-266, 2007.

22. Li F, Li Q, Gao D and Peng Y: The optimal extraction parameters and anti-diabetic activity of flavonoids from Ipomoea batatas leaf. Afr J Tradit Complement Altern Med 6: 195-202, 2009.

23. Li F, Zhang Y and Zhong Z: Antihyperglycemic effect of ganoderma lucidum polysaccharides on streptozotocin-induced diabetic mice. Int J Mol Sci 12: 6135-6145, 2011.
24. Raghavan B and Kumari SK: Effect of Terminalia arjuna stem bark on antioxidant status in liver and kidney of alloxan diabetic rats. Indian J Physiol Pharmacol 50: 133-142, 2006.

25. Ha H, Hwang IA, Park JH and Lee HB: Role of reactive oxygen species in the pathogenesis of diabetic nephropathy. Diabetes Res Clin Pract 82 (Suppl 1): S42-S45, 2008.

26. Cemek M, Kağa S, Simşek N, et al: Antihyperglycemic and antioxidative potential of Matricaria chamomilla L. in streptozotocin-induced diabetic rats. J Nat Med 62: 284-293, 2008. 